\title{
Maximizing the Lifetime of Wireless Devices in Millimeter Wave UAV Networks
}

\author{
Hazim Shakhatreh*, Waed Malkawi \\ Department of Telecommunications Engineering, Hijjawi Faculty for Engineering Technology, Yarmouk University, Irbid 21163, Jordan
}

Received June 5, 2020; Revised July 9, 2020; Accepted July 29, 2020

Copyright $(2020$ by authors, all rights reserved. Authors agree that this article remains permanently open access under the terms of the Creative Commons Attribution License 4.0 International License

\begin{abstract}
The use of unmanned aerial vehicles (UAVs) is growing rapidly across many civil application domains, including providing wireless coverage. Most studies on UAV-based wireless coverage typically consider downlink scenarios from an aerial base station to ground users. The uplink scenario in which ground wireless devices transmit data to an aerial base station is only considered by few studies. However, the frequency bands that are used in these studies are not Millimeter Wave frequency bands, and this limits the applicability of these applications when one needs to consider $5 \mathrm{G}$ networks. In this paper, we are motivated to explore if the placement of UAV can enhance the time durations of uplink transmissions of wireless devices in Millimeter Wave UAV networks. First, we present a realistic Millimeter Wave path loss model and describe the tradeoff introduced by this model. Then, we study the problem of optimal UAV placement, where the objective is to determine the placement of a single UAV such that the sum of time durations of uplink transmissions is maximized. To this end, an algorithm to find the optimal UAV location is proposed. Simulation results are presented to validate the effectiveness of the proposed algorithm.
\end{abstract}

Keywords Unmanned aerial vehicles (UAVs), Millimeter Wave, Lifetime of Wireless Network, Emergency Response, Convex Optimization

1

\section{Introduction}

UAVs can be deployed during emergency situations to provide continuous wireless coverage when the terrestrial network is down [1]. They can also be used to supplement the terrestrial base station to provide users with better coverage and higher data rates [2]. In order to satisfy the requirement of high

\footnotetext{
${ }^{1}$ Part of this work was presented in IEEE IWCMC 2018 [5].
}

throughput in most UAV applications, the potential of integrating Millimeter Wave communications with UAV networks is explored in [3]. There are two main benefits for deploying Millimeter Wave communications to UAV networks. One is that the large available spectrum of Millimeter Wave is able to substantially boost the capacity of UAV networks and hence satisfying the requirement of prompt responses. The other advantage is that the data traffic of UAV networks can be significantly enlarged by using Millimeter Wave since Millimeter Wave communications are capable of offering high throughput in short-range transmissions [3]. The main disadvantage of using a UAV as an aerial base station is their energy capacity; a UAV needs to return periodically to a charging station for recharging process, due to its limited energy capacity. To guarantee the continuous wireless coverage, a UAV will perform a handoff process periodically with one of the additional UAVs available at the charging station [4].

Most studies on UAV-based wireless coverage typically consider downlink scenarios from an aerial base station to ground users. The uplink scenario in which ground wireless devices transmit data to an aerial base station is only considered by few studies. In [5], the authors study the problem of optimal UAV placement, where the objective is to determine the placement of a single UAV such that the sum of time durations of uplink transmissions is maximized. The authors in [6] study the placement problem of UAVs, where the objective is to determine the locations of a set of UAVs that maximize the time duration of uplink transmission until the first wireless device runs out of energy. They also study the problem of minimizing the number of UAVs required to serve the ground users such that the time duration of uplink transmission of each wireless device is greater than or equal to a threshold value. In [7], the problem of UAV placement is studied, where the goal is to find the optimum location of a single UAV that prolongs the lifetime of indoor wireless devices. However, the frequency bands that are used in these studies are not Millimeter Wave frequency bands, and this limits the applicability of these applications when one needs to consider $5 \mathrm{G}$ networks.

In disaster situations (such as earthquakes or floods), be- 
cause of the limited transmitting power of wireless devices, users may not be able to communicate with remote undamaged terrestrial ground stations. They also can not recharge their wireless devices because of physical damage to energy infrastructure. In these cases, it becomes more necessary to provide wireless coverage, as people in the disaster area try to learn about the emergency incident, locate their family and friends, and receive orders to evacuate the region impacted by the disaster $[8,9]$. Note that the energy efficiency at the user device, which is handled by studying the uplink scenario, is of greater importance in a disastrous situation than that at the UAV itself as the UAV can travel long distances to be recharged. In this paper, we are motivated to explore if the placement of UAV can enhance the time durations of uplink transmissions of wireless devices in Millimeter Wave UAV networks. To the authors best knowledge there is no published work that is specifically addressing maximizing the sum of time durations of uplink transmissions under disaster situations in Millimeter Wave UAV networks, where the ground users are unable to recharge their wireless devices due to physical damage to energy infrastructure. We summarize our main contributions as follows:

- We assume a Millimeter Wave path loss model and show the tradeoff introduced by this model.

- We formulate the problem of optimal UAV placement, where the objective is to maximize the lifetime of wireless devices in Millimeter Wave UAV networks, the lifetime represents the sum of time durations of uplink transmissions.

- We prove that the constraint sets of problem can be represented by the intersection of half spheres and the region formed by this intersection is a convex set in terms of two variables.

- We prove that the objective function is a concave function under a restriction on the minimum altitude of UAV and propose a gradient projection-based algorithm to find the optimal location of UAV.

The rest of this paper is organized as follows. In Section 2, we describe the system model. In Section 3, we formulate the problem of UAV placement with an objective of maximizing the sum of time durations of uplink transmissions and present a gradient projection-based algorithm to find the optimal location of the UAV under a restriction on the minimum altitude. Finally, we present our numerical results in Section 4 and make concluding remarks in Section 5.

\section{System Model}

\subsection{System Settings}

Let $\left(X_{u}, Y_{u}, Z_{u}\right)$ denote the 3D location of the UAV. We assume that $|I|$ ground users are located according to a probability distribution $f(x, y)$. Each user $i \in I$ has a wireless device with residual energy $E_{i}$ and the maximum transmit power of each device is $P_{\max }$. The users must be served by a single UAV that acts as aerial base station to collect data from users. We

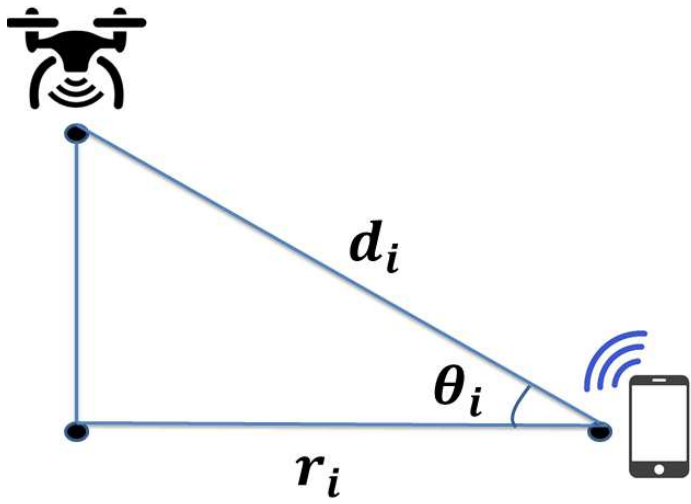

Figure 1. Coverage angle $\theta_{i}$

consider an uplink scenario in which the ground users adopt a frequency division multiple access (FDMA) technique to transmit data to a UAV at a desired data rate $R$. FDMA allocates one subchannel to each user for communications and hence the channels do not interfere with one another. We also assume that each user $i \in I$ is served by a UAV for a time $\tau_{i}$ seconds and this time depends on the residual energy of wireless device represented by the battery level $E_{i}$. The time duration of uplink transmission $\tau_{i}$ must be greater than or equal to $\tau_{t h}$.

\subsection{Path Loss Model}

The path loss models for the Line of Sight (LoS) and nonLine of Sight (nLoS) links at Millimeter Wave frequencies follow [10] and are given as:

$$
\begin{gathered}
L_{L, i}=\alpha_{L}+10 \beta_{L} \log _{10}\left(d_{i}\right), \\
L_{N, i}=\alpha_{N}+10 \beta_{N} \log _{10}\left(d_{i}\right),
\end{gathered}
$$

where $\alpha_{L}, \beta_{L}, \alpha_{N}$, and $\beta_{N}$ are the parameters of the LoS and nLoS path loss models, and $d_{i}=\sqrt{\left(X_{u}-x_{i}\right)^{2}+\left(Y_{u}-y_{i}\right)^{2}+\left(Z_{u}\right)^{2}}$ is the distance between ground user $i$ and a UAV.

In order to account for the human body blockage, we adopt the probability of $\operatorname{LoS}, P_{L}$, for a user $i$ from [11]:

$$
P_{L}\left(\theta_{i}\right)=\exp \left(-\lambda g_{b} \frac{z_{b}-z_{i}}{\tan \left(\theta_{i}\right)}\right)
$$

where $\theta_{i}$ is the coverage angle as shown in Figure $1, \lambda$ is density of human blockers, $g_{b}$ is the diameter of human blockers, $z_{b}$ is the height of human blocker, and $z_{i}$ is the height of receiver.

Then, the average path loss is given by:

$$
L_{a, i}=P_{L}\left(\theta_{i}\right) L_{L, i}+\left[1-P_{L}\left(\theta_{i}\right)\right] L_{N, i}
$$

\subsection{Path Loss Model Tradeoff}

There is a key tradeoff in the Millimeter Wave path loss model when the coverage angle $\left(\theta_{i}\right)$ between the aerial base station and a wireless device changes as shown in Figure 2. At a low altitude, the path loss between the UAV and the ground wireless device decreases, while the probability of line of sight 


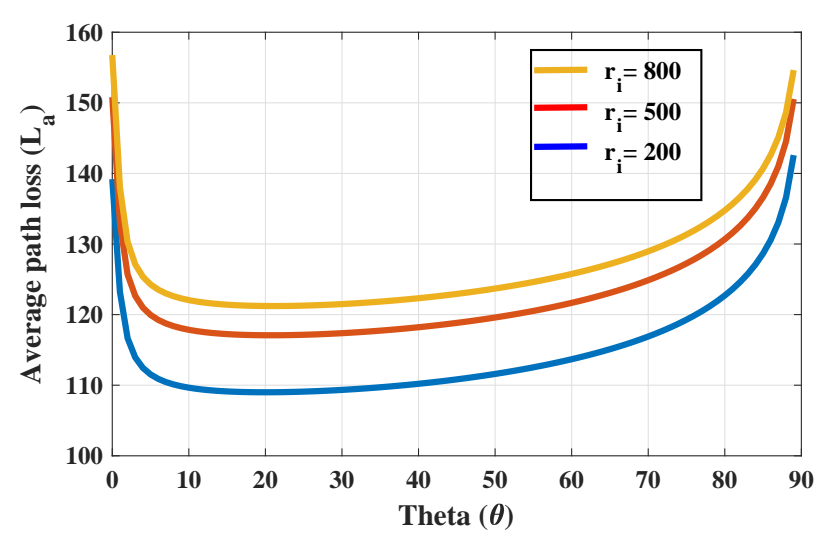

Figure 2. Tradeoff in the path loss model.

links also decreases. On the other hand, at a high altitude line of sight connections exist with a high probability, while the path loss increases.

\section{Problem Formulation}

Consider a transmission between a user located at $\left(x_{i}, y_{i}\right)$ and a UAV located at $\left(X_{u}, Y_{u}, Z_{u}\right)$. The rate for user $i$ is given by:

$$
C_{i}=B_{i} \log _{2}\left(1+\frac{p_{i} / L_{a, i}}{N}\right)
$$

where $B_{i}$ is the transmission bandwidth of user $i, p_{i}$ is the transmit power from user $i$ to the $\mathrm{UAV}, L_{a, i}$ is the average path loss between user $i$ and the UAV and $N$ is the noise power.

Let us assume that all users have the same data rate $R$ and each user has a channel with bandwidth equals $B /|I|$, where $B$ is the UAV bandwidth and $|I|$ is the number of ground users. Then, the minimum power required to satisfy this rate for each user is given by:

$$
p_{i}=\left(2^{\frac{R \cdot|I|}{B}}-1\right) \cdot N \cdot L_{a, i}
$$

Our goal is to find the optimal location of the UAV such that the lifetime of wireless devices defined by $T$ is maximized. Here, the lifetime $T$ represents the sum of time durations of uplink transmissions. Our problem can be formulated as:

$$
\begin{array}{cc}
\max _{\left(X_{u}, Y_{u}, Z_{u}\right), \tau_{i}} T=\sum_{i=1}^{|I|} \tau_{i} & \\
\text { subject to } & \\
\left(2^{\left.\frac{R \cdot|I|}{B}-1\right) \cdot N \cdot L_{a, i} \leq P_{\max }}\right. & \forall i \in I \\
\tau_{i} \geq \tau_{t h} & \forall i \in I \\
\tau_{i} \cdot\left(2^{\left.\frac{R \cdot|I|}{B}-1\right) \cdot N \cdot L_{a, i} \leq E_{i}}\right. & \forall i \in I \\
x_{\min } \leq X_{u} \leq x_{\max } & \\
y_{\min } \leq Y_{u} \leq y_{\max } & \\
z_{\min } \leq Z_{u} \leq z_{\max } &
\end{array}
$$$$
\forall i \in I \quad(6 . a)
$$

Here, constraint set (6.a) ensures that the transmit power of each wireless device should not exceed its maximum transmit power $P_{\max }$. Constraint set (6.b) guarantees that each ground user $i \in I$ is served by UAV for a time greater than $\tau_{t h}$ seconds. Constraint set (6.c) ensures that the total energy consumed by user's device should not exceed its battery energy level $E_{i}$. Constraints (6.d-6.f) represent the minimum and maximum allowed values for $X_{u}, Y_{u}$ and $Z_{u}$.

In this paper, we assume that $z_{\text {min }}$ guarantees $100 \%$ line of sight connection between wireless device $i$ and the UAV. Now, our objective is to find the optimal 2D placement of the UAV such that the lifetime of wireless devices is maximized. Even though the problem has a number of nonlinear constraints, we can transform (6) to an optimization problem with two variables by proving that the constraint sets $(6 . a-6 . c)$ can be represented by the intersection of half spheres and the region formed by this intersection is a convex set in terms of $\left(X_{u}, Y_{u}\right)$.

Theorem 1 The constraint sets (6.a-6.c) can be represented by the intersection of half spheres and the region formed by this intersection is a convex set in terms of $\left(X_{u}, Y_{u}\right)$.

Proof 1 From (3) and (5), the transmit power of ground user $i$ is given by:

$$
p_{i}=\left(2^{\frac{R \cdot|I|}{B}}-1\right) \cdot N \cdot\left(d_{i}^{2} 10^{\frac{\alpha_{L}}{10}}\right)=K d_{i}^{2}
$$

where $K$ is a constant and equals $\left(2^{\frac{R \cdot|I|}{B}}-1\right) . N .\left(10^{\frac{\alpha_{L}}{10}}\right)$. Now, to satisfy constraint set (6.a), $p_{i}$ must be less than $P_{\text {max }}$. From (7), the range of distances $d_{1}$ that satisfies the constraint set (6.a) is given by:

$$
d_{1} \leq \sqrt{\frac{P_{\max }}{K}}
$$

The range of distances $d_{1}$ represents a half sphere with radius $\sqrt{\frac{P_{\max }}{K}}$. To satisfy constraint sets (6.b) and (6.c), $p_{i}$ must be less than $\frac{E_{i}}{\tau_{t h}}$. From (7), the range of distances $d_{2}$ that satisfies constraint sets (6.b) and (6.c) is given by:

$$
d_{2} \leq \sqrt{\frac{E_{i}}{\tau_{t h} K}}
$$

The range of distances $d_{2}$ also represents a half sphere with radius $\sqrt{\frac{E_{i}}{\tau_{t h} K}}$. For each ground user $i$, the transmit power $p_{i}$ and the time duration of uplink transmission $\tau_{i}$ that satisfy the constraint sets (6.a)-(6.c) can be represented by a half sphere with radius:

$$
\min \left\{\sqrt{\frac{P_{\max }}{K}}, \sqrt{\frac{E_{i}}{\tau_{t h} K}}\right\}
$$

The half sphere is a convex set and the intersection of convex sets is also a convex [12].

From Theorem 1, we can represent the transmit power $p_{i}$ and the time duration of uplink transmission $\tau_{i}$ constraints for each 
user as a half sphere. The intersection of all half spheres represents the convex set $V$ that satisfies the constraint sets (6.a)(6.c) for all users. Therefore, we restrict the placement of UAV to be in $V$. Now, we can transform our problem to an optimization problem with two variables $\left(X_{u}, Y_{u}\right)$, where $\left(X_{u}, Y_{u}\right)$ represent the $2 \mathrm{D}$ placement of UAV.

Now, the objective function in (6) can be represented as:

$$
\begin{gathered}
\sum_{i=1}^{|I|} \tau_{i}=\sum_{i=1}^{|I|} \frac{E_{i}}{p_{i}}=\sum_{i=1}^{|I|} \frac{E_{i}}{\left(2^{\frac{R \cdot|I|}{B}}-1\right) \cdot N \cdot L_{a, i}}= \\
\sum_{i=1}^{|I|} \frac{E_{i}}{\left(2^{\frac{R \cdot|I|}{B}}-1\right) \cdot N \cdot\left(d_{i}^{2} 10^{\frac{\alpha_{L}}{10}}\right)}=\sum_{i=1}^{|I|} \frac{E_{i}}{K d_{i}^{2}}
\end{gathered}
$$

Since $K$ is constant, our problem can be formulated as:

$$
\begin{gathered}
\max _{\left(X_{u}, Y_{u}\right)} \sum_{i=1}^{|I|} \frac{E_{i}}{\left(X_{u}-x_{i}\right)^{2}+\left(Y_{u}-y_{i}\right)^{2}+z_{\text {min }}^{2}} \\
\text { subject to } \\
\left(X_{u}, Y_{u}\right) \in \\
\bigcap_{i=1}^{|I|}\left\{\left(X_{u}, Y_{u}\right) \in \mathbf{R}^{2} \mid \sqrt{\left(X_{u}-x_{i}\right)^{2}+\left(Y_{u}-y_{i}\right)^{2}+z_{\text {min }}^{2}} \leq d_{i}\right\}
\end{gathered}
$$

The transformed problem (12) is equivalent to problem (6). In the next theorem, we prove that the objective function is concave under a restriction on the coverage angle of ground user $\theta_{i}$. The coverage angle is shown in Figure 1 and depends on the $3 \mathrm{D}$ placement of the UAV and the $2 \mathrm{D}$ location of ground user. This theorem enables us to find the optimal placement for the UAV.

Theorem 2 The objective function of (12) is concave if the coverage angle of each wireless device is greater that $60^{\circ}$.

Proof 2 We know that the nonnegative weighted sums preserve the concavity of function [12]. Since $E_{i}>0, \forall i \in I$, we need to prove that (13) is a concave function.

$$
f=\frac{1}{\left(X_{u}-x_{i}\right)^{2}+\left(Y_{u}-y_{i}\right)^{2}+z_{\text {min }}^{2}}, \forall i \in I
$$

Using the second order condition, the function $f$ is concave if the Hessian is negative semidefinite [12]. Now, the Hessian is negative semidefinite if we satisfy these conditions:

$$
\begin{gathered}
\text { (a) } \frac{d^{2} f}{d X_{u}^{2}} \leq 0, \quad \forall i \in I \\
(b) \frac{d^{2} f}{d Y_{u}^{2}} \leq 0, \quad \forall i \in I \\
\text { (c) } \frac{d^{2} f}{d X_{u}^{2}} \frac{d^{2} f}{d Y_{u}^{2}}-\left(\frac{d^{2} f}{d X d Y_{u}}\right)^{2} \geq 0, \quad \forall i \in I
\end{gathered}
$$

To check the first condition, we need to find $\frac{d^{2} f}{d X_{u}^{2}}$ :

$$
\begin{gathered}
\frac{d f}{d X_{u}}=\frac{-2\left(X_{u}-x_{i}\right)}{\left(\left(X_{u}-x_{i}\right)^{2}+\left(Y_{u}-y_{i}\right)^{2}+z_{\text {min }}^{2}\right)^{2}} \\
\frac{d^{2} f}{d X_{u}^{2}}=\frac{-2\left(\left(X_{u}-x_{i}\right)^{2}+\left(Y_{u}-y_{i}\right)^{2}+z_{\text {min }}^{2}\right)^{2}}{\left(\left(X_{u}-x_{i}\right)^{2}+\left(Y_{u}-y_{i}\right)^{2}+z_{\text {min }}^{2}\right)^{4}}+ \\
\frac{8\left(X_{u}-x_{i}\right)^{2}\left(\left(X_{u}-x_{i}\right)^{2}+\left(Y_{u}-y_{i}\right)^{2}+z_{\text {min }}^{2}\right)}{\left(\left(X_{u}-x_{i}\right)^{2}+\left(Y_{u}-y_{i}\right)^{2}+z_{\text {min }}^{2}\right)^{4}} \\
=\frac{-2\left(\left(X_{u}-x_{i}\right)^{2}+\left(Y_{u}-y_{i}\right)^{2}+z_{\text {min }}^{2}\right)+8\left(X_{u}-x_{i}\right)^{2}}{\left(\left(X_{u}-x_{i}\right)^{2}+\left(Y_{u}-y_{i}\right)^{2}+z_{\text {min }}^{2}\right)^{3}} \\
=\frac{6\left(X_{u}-x_{i}\right)^{2}-2\left(Y_{u}-y_{i}\right)^{2}-2 z_{\min }^{2}}{\left(\left(X_{u}-x_{i}\right)^{2}+\left(Y_{u}-y_{i}\right)^{2}+z_{\text {min }}^{2}\right)^{3}}
\end{gathered}
$$

From (15), $\frac{d^{2} f}{d X_{u}^{2}} \leq 0, \forall i \in I$ if:

$$
z_{\text {min }}^{2}>3\left(X_{u}-x_{i}\right)^{2}-\left(Y_{u}-y_{i}\right)^{2}, \forall i \in I
$$

Similarly, $\frac{d^{2} f}{d Y_{u}^{2}} \leq 0, \forall i \in I$ if:

$$
z_{\text {min }}^{2}>3\left(Y_{u}-y_{i}\right)^{2}-\left(X_{u}-x_{i}\right)^{2}, \forall i \in I
$$

To check the third condition, we need to find $\frac{d^{2} f}{d X_{u}^{2}} \frac{d^{2} f}{d Y_{u}^{2}}-$ $\left(\frac{d^{2} f}{d X d Y_{u}}\right)^{2}$ :

$$
\frac{d^{2} f}{d X_{u} d Y_{u}}=\frac{8\left(X_{u}-x_{i}\right)\left(Y_{u}-y_{i}\right)}{\left(\left(X_{u}-x_{i}\right)^{2}+\left(Y_{u}-y_{i}\right)^{2}+z_{\text {min }}^{2}\right)^{3}}
$$

From (18), we get:

$$
\begin{gathered}
\frac{d^{2} f}{d X_{u}^{2}} \frac{d^{2} f}{d Y_{u}^{2}}-\left(\frac{d^{2} f}{d X d Y_{u}}\right)^{2}= \\
\left(\frac{-2\left(\left(X_{u}-x_{i}\right)^{2}+\left(Y_{u}-y_{i}\right)^{2}+z_{\text {min }}^{2}\right)+8\left(X_{u}-x_{i}\right)^{2}}{\left(\left(X_{u}-x_{i}\right)^{2}+\left(Y_{u}-y_{i}\right)^{2}+z_{\text {min }}^{2}\right)^{3}} \times\right. \\
\left.\frac{-2\left(\left(X_{u}-x_{i}\right)^{2}+\left(Y_{u}-y_{i}\right)^{2}+z_{\text {min }}^{2}\right)+8\left(Y_{u}-y_{i}\right)^{2}}{\left(\left(X_{u}-x_{i}\right)^{2}+\left(Y_{u}-y_{i}\right)^{2}+z_{\text {min }}^{2}\right)^{3}}\right)- \\
\frac{64\left(X_{u}-x_{i}\right)^{2}\left(Y_{u}-y_{i}\right)^{2}}{\left(\left(X_{u}-x_{i}\right)^{2}+\left(Y_{u}-y_{i}\right)^{2}+z_{\text {min }}^{2}\right)^{6}}= \\
\frac{4\left(\left(X_{u}-x_{i}\right)^{2}+\left(Y_{u}-y_{i}\right)^{2}+z_{\text {min }}^{2}\right)^{2}}{\left(\left(X_{u}-x_{i}\right)^{2}+\left(Y_{u}-y_{i}\right)^{2}+z_{\text {min }}^{2}\right)^{6}}- \\
\frac{16\left(Y_{u}-y_{i}\right)^{2}\left(\left(X_{u}-x_{i}\right)^{2}+\left(Y_{u}-y_{i}\right)^{2}+z_{\text {min }}^{2}\right)}{\left(\left(X_{u}-x_{i}\right)^{2}+\left(Y_{u}-y_{i}\right)^{2}+z_{\text {min }}^{2}\right)^{6}}- \\
\frac{16\left(X_{u}-x_{i}\right)^{2}\left(\left(X_{u}-x_{i}\right)^{2}+\left(Y_{u}-y_{i}\right)^{2}+z_{m i n}^{2}\right)}{\left(\left(X_{u}-x_{i}\right)^{2}+\left(Y_{u}-y_{i}\right)^{2}+z_{\text {min }}^{2}\right)^{6}}+
\end{gathered}
$$




$$
\begin{gathered}
\frac{64\left(X_{u}-x_{i}\right)^{2}\left(Y_{u}-y_{i}\right)^{2}}{\left(\left(X_{u}-x_{i}\right)^{2}+\left(Y_{u}-y_{i}\right)^{2}+z_{\text {min }}^{2}\right)^{6}}- \\
\frac{64\left(X_{u}-x_{i}\right)^{2}\left(Y_{u}-y_{i}\right)^{2}}{\left(\left(X_{u}-x_{i}\right)^{2}+\left(Y_{u}-y_{i}\right)^{2}+z_{\text {min }}^{2}\right)^{6}}= \\
\frac{4\left(\left(X_{u}-x_{i}\right)^{2}+\left(Y_{u}-y_{i}\right)^{2}+z_{\text {min }}^{2}\right)}{\left(\left(X_{u}-x_{i}\right)^{2}+\left(Y_{u}-y_{i}\right)^{2}+z_{\text {min }}^{2}\right)^{5}}+ \\
\frac{-16\left(X_{u}-x_{i}\right)^{2}-16\left(Y_{u}-y_{i}\right)^{2}}{\left(\left(X_{u}-x_{i}\right)^{2}+\left(Y_{u}-y_{i}\right)^{2}+z_{\text {min }}^{2}\right)^{5}}= \\
\frac{-12\left(X_{u}-x_{i}\right)^{2}-12\left(Y_{u}-y_{i}\right)^{2}+4 z_{\text {min }}^{2}}{\left(\left(X_{u}-x_{i}\right)^{2}+\left(Y_{u}-y_{i}\right)^{2}+z_{\text {min }}^{2}\right)^{5}} \\
\text { From }(19), \frac{d^{2} f}{d X_{u}^{2}} \frac{d^{2} f}{d Y_{u}^{2}}-\left(\frac{d^{2} f}{d X d Y_{u}}\right)^{2} \geq 0, \forall i \in I \text { if: } \\
z_{\text {min }}^{2}>3\left(X_{u}-x_{i}\right)^{2}+3\left(Y_{u}-y_{i}\right)^{2}, \forall i \in I
\end{gathered}
$$

From (16), (17) and (20), the Hessian is negative semidefinite if we satisfy these conditions:

$$
\begin{gathered}
(a) z_{\text {min }}^{2}>3\left(X_{u}-x_{i}\right)^{2}-\left(Y_{u}-y_{i}\right)^{2}, \forall i \in I \\
(b) z_{\text {min }}^{2}>3\left(Y_{u}-y_{i}\right)^{2}-\left(X_{u}-x_{i}\right)^{2}, \forall i \in I \\
(c) z_{\text {min }}^{2}>3\left(X_{u}-x_{i}\right)^{2}+3\left(Y_{u}-y_{i}\right)^{2}, \forall i \in I
\end{gathered}
$$

From the three conditions in (21), we can notice that if we satisfy condition (c), we then satisfy conditions $(a)$ and $(b)$. Let us define $d_{\max }$ as a maximum possible $2 D$ distance in the geographical area (i.e., if the users are distributed in a circular geographical area, then $d_{\max }$ is equal to the diameter of circle). From condition (c), if $z_{\min }>\sqrt{3} d_{\max }$ then the objective function of (12) is concave where $d_{\max }>$ $\sqrt{\left(X_{u}-x_{i}\right)^{2}+\left(Y_{u}-y_{i}\right)^{2}}, \forall i \in I$. Then, the coverage angle $\theta_{i}$ must be greater than or equal to $\tan ^{-1} \frac{\sqrt{3} d_{\max }}{d_{\max }}=60^{\circ}$.

Here, we can notice that the altitude of UAV $z_{\text {min }}$ controls the concavity of the objective function. Theorem 2 enables us to find the optimal placement for the UAV, when the coverage angle $\theta_{i}$ of each wireless device is greater than or equal to $60^{\circ}$.

For ground-to-air communications, each device will typically have a line of sight view towards a specific UAV with a given probability. This line of sight probability depends on the environment [13], location of the device and the UAV, and the elevation angle between the device and the UAV [14]. To verify that the coverage angle that we characterize in Theorem 2 guarantees a line of sight path, we utilize Line of Sight probability model for downlink scenario. In Figure 3, we plot the probability of having a LOS wireless connection. We can notice that the coverage angle that we characterize in Theorem 2 gives us 100\% LOS probability. Therefore, our assumption that a wireless channel is a line of sight dominated is a realistic assumption.

Now, we propose to use the Gradient Projection Algorithm [15] to find the optimal placement of a UAV, when the UAV's altitude satisfies the condition in Theorem 2. The gradient projection algorithm is given by:

$$
\left.\left(X_{u}, Y_{u}\right)^{n+1}=\left[\left(X_{u}, Y_{u}\right)^{n}+\gamma \cdot \nabla F\left(\left(X_{u}, Y_{u}\right)^{n}\right)\right)\right]^{+} .
$$

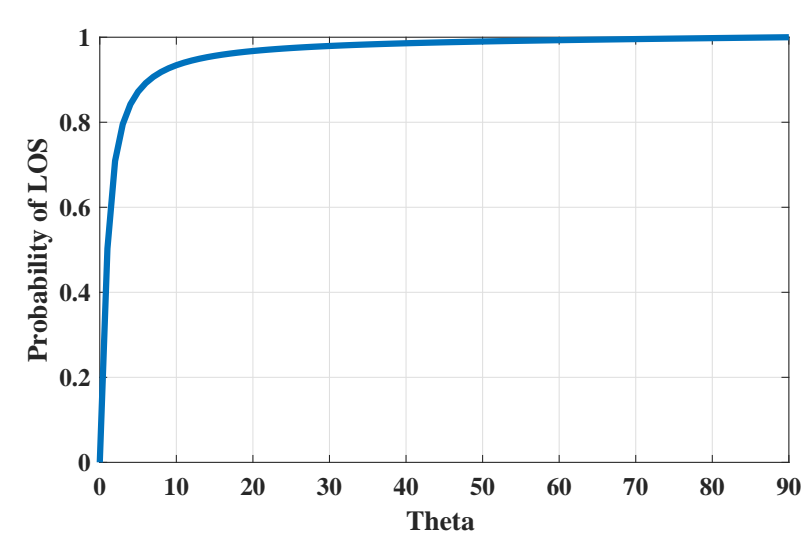

Figure 3. Probability line of sight.

Here, $n$ is the iteration number, $\gamma$ is a positive step size, $\nabla F$ is the gradient of the objective function in (12) and $[q]^{+}$denotes the orthogonal projection of vector $q$ onto convex set $Q$. In particular, $[q]^{+}$is defined by: .

$$
[q]^{+}=\arg \min _{w \in Q}\|w-q\|_{2}
$$

The pseudo code of the gradient projection algorithm is shown in Algorithm 1.

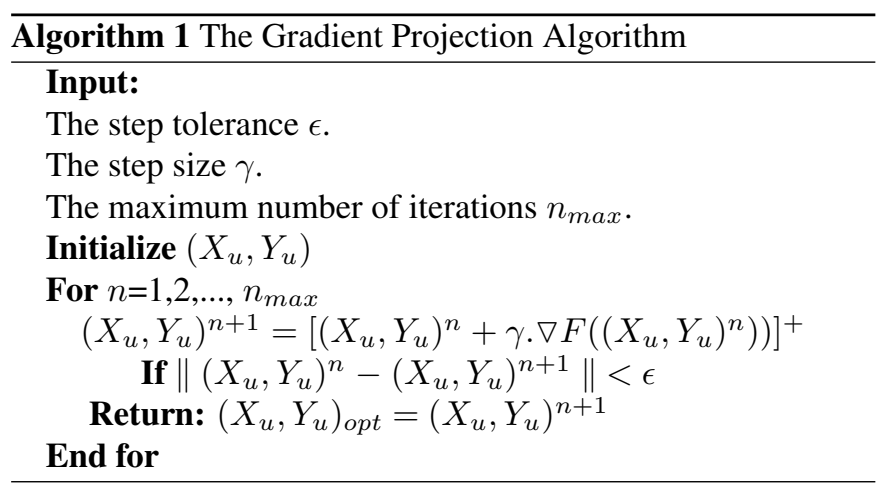

\section{Numerical Results}

In this section, we first verify the results of Theorem 2, then we use the gradient projection algorithm to find the optimal placement for UAV under different cases. Table 1 lists the parameters used in the numerical analysis.

To verify the results of Theorem 2 , we assume that 100 wireless devices are uniformly distributed in a geographical area of size $100 m \times 100 m$, then we plot the objective function in (12) without any constraints at two different altitudes of the UAV. The first value for altitude $z_{\min }$ is 500 meters, which is greater than $\sqrt{3} d_{\max }$ and satisfies the condition in Theorem 2 . The second value for altitude is 10 meters and it does not satisfy the condition in Theorem 2. In Figure 4.a, we can notice that the objective is concave when the altitude of UAV is equal to 500 meters. On the other hand, the objective function becomes non-concave at 10 meters as shown in Figure 4.b. We can also notice that the objective function at low altitude has better costs 


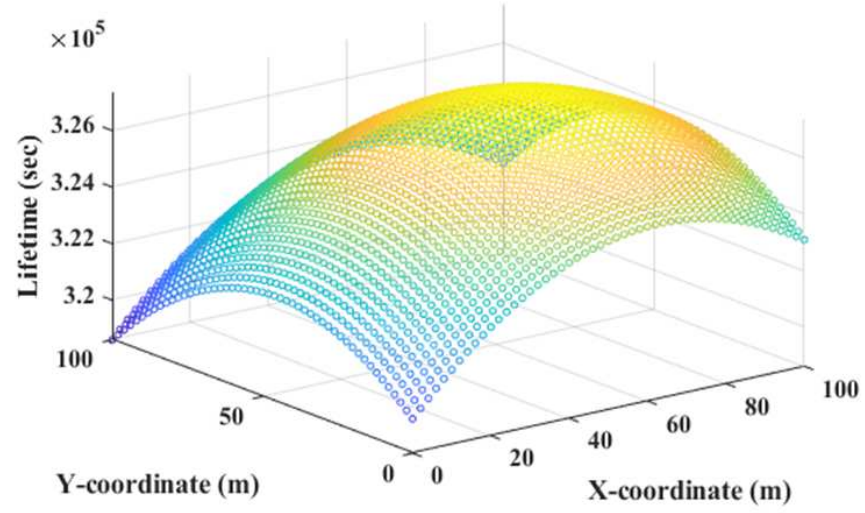

(a) Lifetime at 500 meters

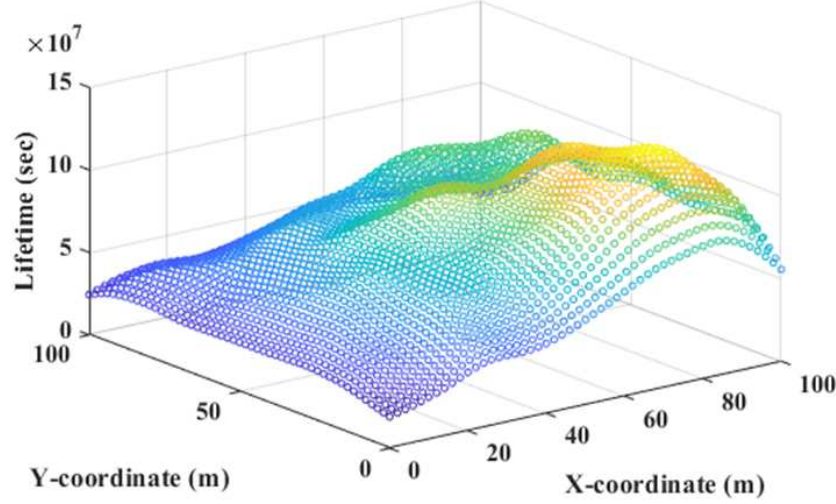

(b) Lifetime at 10 meters

Figure 4. Lifetime of wireless devices at different altitudes.

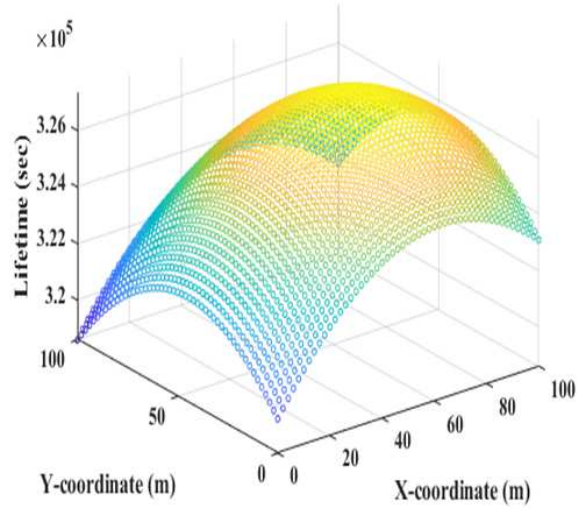

(a) Total cost

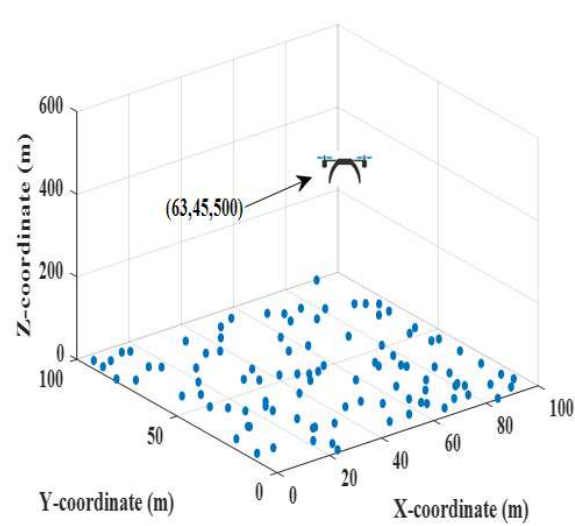

(b) 3D placement of UAV

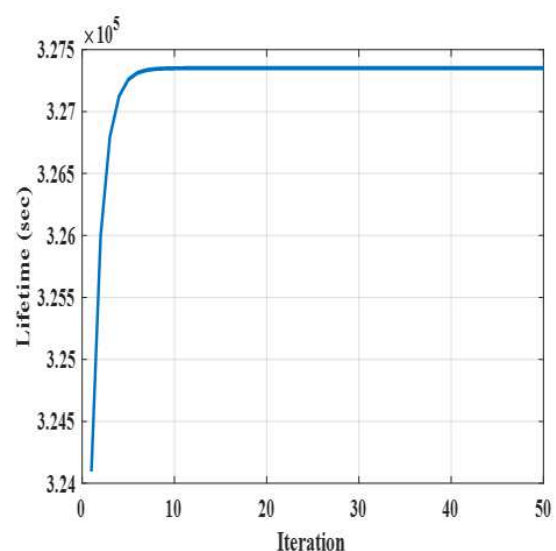

(c) Convergence speed of algorithm

Figure 5. Simulation results of the uniform distribution case.

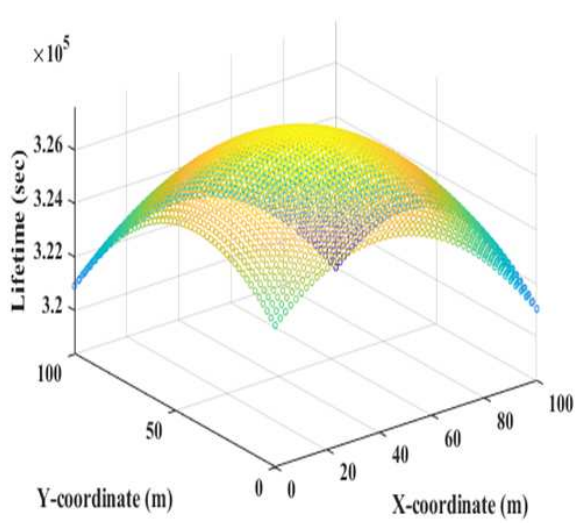

(a) Total cost

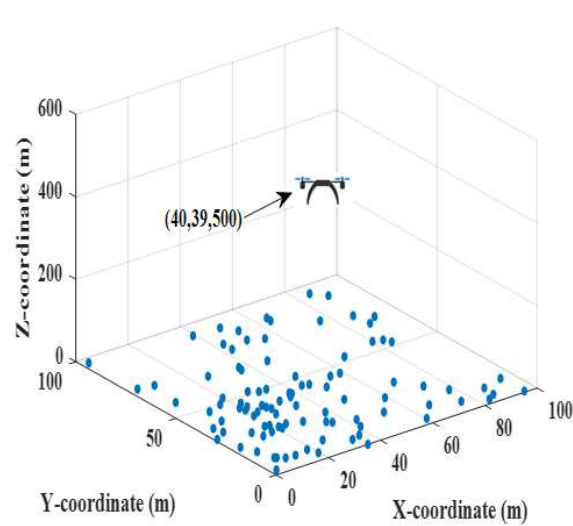

(b) 3D placement of UAV

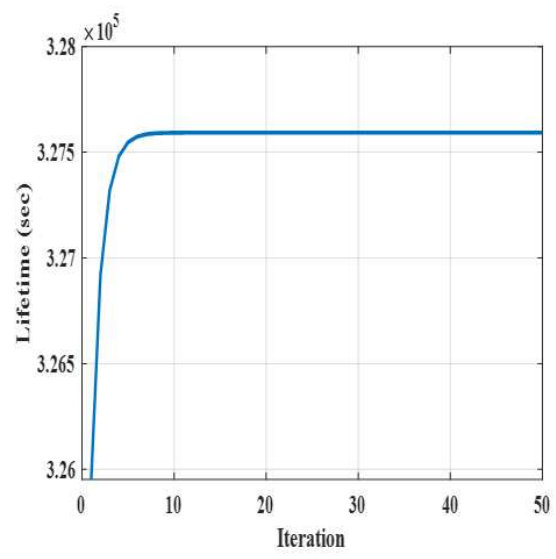

(c) Convergence speed of algorithm

Figure 6. Simulation results of the non-uniform distribution case. 
Table 1. Parameters in numerical analysis

\begin{tabular}{|c|c|}
\hline Dimensions of area & {$[0,100] \times[0,100]$} \\
\hline Number of ground users & 100 users \\
\hline Maximum number of iterations $n_{\max }$ & 50 \\
\hline Maximum transmit power of wireless device $P_{\max }$ & $0.5 \mathrm{watt}$ \\
\hline Energy of each wireless device $E_{i}$ in joule & $4500+13500^{*} \mathrm{rand}(100,1)$ \\
\hline Data rate $R$ & $100 \mathrm{Mbps}$ \\
\hline Total bandwidth $B$ & $1 \mathrm{GHz}$ \\
\hline Noise Power $N$ & $1 \times 10^{-14}$ \\
\hline Carrier frequency $f$ & $28 \mathrm{Ghz}$ \\
\hline Threshold time duration of uplink transmission $\tau_{t h}$ & $900 \mathrm{~seconds}$ \\
\hline Minimum altitude for UAV & $500 \mathrm{~meters}$ \\
\hline Height of a human blocker $z_{b}$ & $1.7 \mathrm{~meter}$ \\
\hline Diameter of a human blocker $g_{b}$ & $0.5 \mathrm{~meter}$ \\
\hline Density of human blockers $\lambda$ & $0.06 \mathrm{blockers} / \mathrm{m}^{2}$ \\
\hline$\alpha_{L}$ & 61.4 \\
\hline
\end{tabular}

compared to results at high altitude, which make our approach practical for UAVs that have high altitude constraints.

In Figure 5, we place the UAV at 500 meters and use the gradient projection algorithm to find the optimal 2D placement that maximizes the lifetime of wireless devices when the ground users are uniformly distributed. The optimal placement for $\operatorname{UAV}$ is $(63,45,500)$ and the optimal lifetime is $3.2735 \times 10^{5}$ seconds. We can notice that the projection of the optimal point is located near the center of deployment region. This is because the devices that have minimum costs are located at the corners of the deployment region and placing the projection of UAV near the center maximizes the lifetime. In Figure 6, we use the gradient projection algorithm to find the optimal 2D placement when the ground users are nonuniformly distributed. The optimal placement for UAV is (40, 39,500 ) and the optimal lifetime is $3.2759 \times 10^{5}$ seconds. We can notice that the placement of UAV is near the high density region.

\section{Conclusions}

In this paper, the problem of optimal UAV placement in Millimeter Wave UAV networks is studied. First, we present a realistic Millimeter Wave path loss model and describe the tradeoff introduced by this model. Then, we study the problem of optimal UAV placement, where the objective is to determine the placement of a single UAV such that the sum of time durations of uplink transmissions is maximized. To this end, an algorithm to find the optimal UAV location is proposed. Simulation results are presented to validate the effectiveness of the proposed algorithm. In future work, we will consider the effect of buildings and NLOS links. Also, we will study the problem using multiple UAVs.

\section{REFERENCES}

[1] H. Shakhatreh, A. Khreishah, J. Chakareski, H. Bany Salameh, and I. Khalil. On the continuous coverage problem for a swarm of UAVs. 2016 IEEE 37th Sarnoff Symposium, 130-135, 2016.

[2] H. Shakhatreh, A. Sawalmeh, A. Al-Fuqaha, Z. Dou, , E. Almaita, I. Khalil, N. Othman, A. Khreishah, and M. Guizani.
Unmanned Aerial Vehicles: A Survey on Civil Applications and Key Research Challenges. IEEE Access, 48572-48634, 2019.

[3] W. Yi, Y. Liu, Y. Deng, A. Nallanathan. Clustered UAV Networks with Millimeter Wave Communications: A Stochastic Geometry View. IEEE Transactions on Communications, 2020.

[4] H. Shakhatreh, A. Khreishah, and I. Khalil. Indoor Mobile Coverage Problem Using UAVs. IEEE Systems Journal, 3837-3848, 2018.

[5] H. Shakhatreh, A. Khreishah. Optimal Placement of a UAV to Maximize the Lifetime of Wireless Devices. 14th International Wireless Communications and Mobile Computing Conference (IWCMC), 1225-1230, 2018.

[6] H. Shakhatreh, A. Khreishah, B. Ji. UAVs to the Rescue: Prolonging the Lifetime of Wireless Devices Under Disaster Situations. IEEE Transactions on Green Communications and Networking, 942-954, 2019.

[7] H. Shakhatreh. Optimal Placement of a UAV to Prolong the Lifetime of Indoor Wireless Devices. Universal Journal of Electrical and Electronic Engineering, 108-114, 2019.

[8] T. Taniguchi, Y. Karasawa, N. Nakajima. Effect of Coordinated Base Station in Uplink Transmission Under Disaster Cells. IEEE International Conference on Selected Topics in Mobile and Wireless Networking, 83-88, 2012.

[9] T. Simon, A. Goldberg, B. Adini. Socializing in Emergencies-A Review of the Use of Social Media in Emergency Situations. International Journal of Information Management, 609$619,2015$.

[10] M.R. Akdeniz, Y. Liu, M.K. Samimi, S. Sun, S. Rangan, T.S. Rappaport, E. Erkip. Millimeter Wave Channel Modeling and Cellular Capacity Evaluation. IEEE journal on selected areas in communications, 1164-1179, 2014.

[11] M. Gapeyenko, I. Bor-Yaliniz, S. Andreev, H. Yanikomeroglu, Y. Koucheryavy. Effects of Blockage in Deploying mmWave Drone Base Stations for 5G Networks and Beyond. IEEE International Conference on Communications Workshops (ICC Workshops), 1-6, 2018.

[12] S. Boyd, L. Vandenberghe. Convex optimization. Cambridge university press, 2004.

[13] I. Bor-Yaliniz, S. S. Szyszkowicz, H. Yanikomeroglu. Environment-Aware Drone-Base-Station Placements in Modern 
Metropolitans. IEEE Wireless Communications Letters, 372375, 2017.

[14] M. Mozaffari, W. Saad, M. Bennis, M. Debbah. Mobile Internet of Things: Can UAVs Provide an Energy-Efficient Mobile Architecture?. IEEE global communications conference (GLOBECOM), 1-6, 2016.

[15] DP. Bertsekas, JN. Tsitsiklis. Parallel and Distributed Computation: Numerical Methods. Prentice hall Englewood Cliffs, NJ, 1989. 